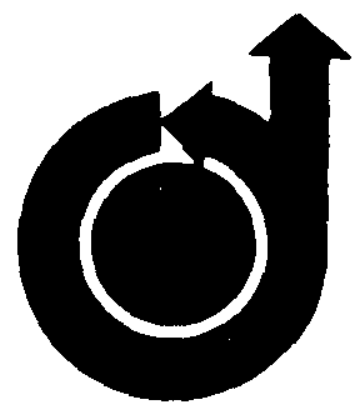

78-247

\title{
Large Amplitude Shock Wave Motion in Two Dimensional Transonic Channel Flow
}

T.C. Adamson, Jr., A.F. Messiter and M.S. Liou, University of Michigan, Ann Arbor, Mich.

\section{AIAA 16TH AEROSPACE SCIENCES MEETING}

Huntsville, Alabama/January 16-18, 1978

For permission to copy or republish, contact the Amerlcan Institute of Aeronautics and Astronautics, 1290 Avenue of the Americas, New York, N.Y. 10019. 


\title{
IN TWO DIMENSIONAL TRANSONIC CHANNEL FLOWS ${ }^{\dagger}$
}

\author{
T. C. Adamson, Jr. , * A. F. Messiter, ** and M. S. Liou ${ }^{* * * * *}$ \\ The University of Michigan \\ Ann Arbor, Michigan 48109
}

\section{Abstract}

Two dimensional unsteady transonic channel flow with a shock wave is considered for the slowly varying time regime. Pressure oscillations, introduced downstream of the shock wave, cause the shock wave to oscillate; the case considered is that where the shock is forced upstream of the throat, disappears, and then reappears as the downstream pressure first increases and then decreases. The subsequent shock wave motion con sists of oscillations either about the throat or about the original steady flow shock position, depending upon parametric relationships developed in the analysis. These two cases and the dividing case are illustrated with example calculations.

\section{Introduction}

Recent papers on unsteady transonic channel flows, where the unsteadiness arises as a result of pressure oscillations introduced downstream of a shock wave, either have dealt with relatively small amplitude shock oscillations ${ }^{1,2}$, or have included only very brief discussions of possible large amplitude oscillations. ${ }^{3}$ Thus, if the channel half width at the throat is $\bar{L}$ loverbars denote dimensional quantities), $\bar{a}^{-*}$ is the critical sound speed, and $\epsilon$ is a small parameter which measures the typical difference between the fluid velocity and the sound speed, the case where the impressed pressure oscillations have amplitude $O\left(\epsilon^{2}\right)$ with a period $O(\epsilon)$, and the amplitude of the shock-wave oscillation is $O(\epsilon)$, is covered in reference 1 ; solutions are presented for a symmetric channel. In reference 2, where a relatively highly curved asymmetric channel is considered, the impressed pressure oscillations have amplitude $O\left(\epsilon^{2}\right)$ and now a period of $O\left(\epsilon^{2}\right)$, so the amplitude of the shock oscillations is $O(1)$. However, only a relatively small amplitude is actually considered, the emphasis being on the asymmetry of the flow. Finally, in reference 3, several combinations of the impressed pressure amplitude and period are dis cussed, and it is pointed out by means of a simple example that it is possible to analyze the case where the shock wave moves upstream to the throat, disappears, and then later reappears. In each of the above named references, a "slowly varying "time regime is considered, where if the characteristic time associated with the imposed

${ }^{T}$ This work was partially supported by Naval Air Systems Command under Contract N0019-76-C0435. This support is gratefully acknowledged. * Professor of Ae rospace Enginee ring, Associate Fellow, AIAA.

**P Professor of Aerospace Engineering, Member, AIAA.

**;:k Re search Investigator, Student Member, AIAA. CCopyright 1978 by the authors, with release to AIAA to publish in all forms. flow disturbance is $O\left(\overline{\mathrm{T}}_{\mathrm{ch}}\right)$, then $\overline{\mathrm{T}}_{\mathrm{ch}} \gg \overline{\mathrm{L}} / \overline{\mathrm{a}}^{* *}$

The present paper is concerned with the case where the pressure oscillation has amplitude $O\left(e^{2}\right)$ and period $O\left(\epsilon^{2}\right), \cdots$ again in the slowly varying time regime, so that, as will be seen, the shock-wave oscillation amplitude is $O(1)$. In particular, we consider those cases where, as a result of the pressure oscillations introduced downstream, the shock wave moves upstream of the throat, disappears, and then reappears as the downstream pressure decreases. Then, depending upon the flow parameters, it will be shown that the subsequent shock motion will follow one of several different paths, ranging from an oscillation in which the shock disappears and reappears on a periodic basis to a periodic oscillation during which the shock never again reaches the throat. The possible applications to unsteady flow problems in inlets and flows between blades in turbomachinery indicate that the solutions to be presented for simple boundary shapes will be quite helpful toward understanding flows in more complex geometries.

\section{Derivation of Equation for Shock-Wave Position}

The problem considered is that of a symmetric two-dimensional channel with an arbitrary wall shape, in which the re is a transonic flow with a shock wave. Coordinates $x$ and $y$, with corresponding velocity components $u$ and $v$, are aligned parallel and perpendicular to the channel axis, respectively. The flow upstream of the wave is steady; pressure oscillations are impressed upon the flow downstream of the shock, at $x=x$, say, causing the shock wave to oscillate. The gas is assumed to follow the perfect gas law and to have constant specific heats. The flow upstream of the shock wave is isentropic, and because the flow is transonic, the shock is weak enough that a velocity potential may be used to the order desired.

The coordinates $x$ and $y$ are made dimensionless with respect to $\bar{L}$, the time $T$ with respect to $\bar{L} / \bar{a} *$, and velocities with respect to $\mathrm{a}^{*}$; hence, the dimensionless velocity potential is referred to the product $\overline{\mathrm{L}}_{\widetilde{\mathrm{a}}}^{*}$. The pressure $P$, density $\rho$, and temperature $\widetilde{T}$, are made dimensionless with respect to their critical values, and the enthalpy is referred to $\bar{a} * 2$.

The wall shapes considered are written as follows, for symmetric channels:

$$
\mathrm{y}_{\mathrm{w}}= \pm\left(1+\epsilon^{2} \mathrm{f}(\mathrm{x})\right)
$$

where $f(x)$ is the arbitrary wall shape function, such that $f(0)=f^{\prime}(0)=0$. Thus, $x$ is measured from the channel throat. The radius of curvature of the channel at the throat is $O\left(\epsilon^{-2}\right)$, from eqn. (1), and as will be seen later, $u-1=O(\epsilon)$; for transonic flow $\epsilon \ll 1$. 
Unsteady flows may be characterized by prescribing the relative order of the characteristic time associated with the impressed disturbances, $\overline{\mathrm{T}}_{\mathrm{ch}}$, and the characteristic time associated with the acoustic waves traveling through the channel, $\overline{\mathrm{L}} / \overline{\mathrm{a}}^{*}$. As mentioned previously, the slowly varying time regime is considered here, where $\overline{\mathrm{T}}_{\mathrm{L}} \gg \overline{\mathrm{a}}^{\mathrm{k}}$. Therefore, a parameter $\tau$ is introduced and a new stretched time coordinate is made nondimensional with $\overline{\mathrm{T}}_{\mathrm{ch}}$ as follows:

$$
\mathrm{r} \equiv \overline{\mathrm{T}}_{\mathrm{ch}} /\left(\overline{\mathrm{L}} / \mathrm{a}^{-*}\right) \quad \mathrm{T}=\tau \mathrm{t}
$$

where $\tau \gg 1$ and $t=O(1)$. The relationship be tween $\tau$ and $\epsilon$ depends upon the case considered. Since $u-1=O(\epsilon)$, the time $T_{s h}$ required for a signal to travel upstream from the origin of the flow disturbance to the shock wave is $O\left(\overline{\mathrm{L}} / \bar{a}^{-*} \in\right)$, and so $\mathrm{T}_{\mathrm{ch}} / \overline{\mathrm{T}}_{\mathrm{sh}}=O(\epsilon \tau)$. If $\tau=O\left(\epsilon^{-1}\right)$, the case studied in reference $I$, then $\bar{T}_{s h}=O\left(\bar{T}_{c h}\right)$ and there is a lag between the impressed oscillations in pressure and the pressure, temperature, etc., oscillations in the channel now downstream of the shock. If $\tau=O\left(\epsilon^{-2}\right)$, the case studied here and in reference 2 , pressure signals from downstream of the shock wave reach the shock "instantaneously" compared with the period of the impressed changes in pressure; i.e., $\overline{\mathrm{T}}_{\mathrm{sh}} / \overline{\mathrm{T}}_{\mathrm{ch}}=\mathrm{O}(\epsilon)$. As will be seen, this leads to shock-wave oscillation amplitudes of order one. Thus, we choose

$$
\tau=\left(k \epsilon^{2}\right)^{-1}
$$

where $k$ is an arbitrary constant of order unity.

The general method of solution follows that used in references 1 and 2 , The solutions for $u, v, P, \rho$, and $\widetilde{T}$ are written in the form of asymptotic expansions for $x, y, t=O(1)$. Since the flow upstream of the shock is steady and the im pressed pressure downstream of the wave has time variations only in second order terms, then to first order (i.e., $O(\epsilon)$ ), the flow is steady and the time dependence enters only in second order terms. As shown previously ${ }^{3,2}$ and as illustrated in figure 1 , variations in pressure of order $\epsilon^{2}$ at $\mathrm{x}=\mathrm{X}$ are sufficient to cause local pressure variations of order $\epsilon$ across the shock; as will be seen, these variations are also sufficient to cause shock wave displacements of order one. Hence, relatively small impressed pressure variations can cause large local pressure changes over a large part of the channel; this, of course, is a very important element in the study of blade flutter in transonic turbomachinery.

The only difference between the problem considered in reference $I$ and that studied here is that in reference $\mathbf{l}$, hereafter referred to as case (1), $\tau=O\left(\epsilon^{-1}\right)$ whereas in the present case, $\tau=O\left(\epsilon^{-2}\right)$; the radius of curvature of the channel at the throat, $O\left(\epsilon^{-2}\right)$, and the order of the impressed oscillations in the pressure are the same. Hence, in the general governing equations, since

$$
\partial / \partial \mathrm{T}=\frac{1}{\tau}(\partial / \partial t)
$$

it is seen that in the present case the partial derivatives with respect to time will be one order higher than they were in case (1). Therefore, the general outer solutions may be derived easily from the outer solutions given in reference $1, i, e$, those solutions, valid outside a thin region enclosing the shock wave. Because the solutions in question do not satisfy the shock wave jump conditions in second order, it is necessary here also to consider an inner region about the shock, in which the solutions satisfy the jump conditions at the shock and match with these outer solutions in the appropriate limit as the outer regions are approached. Then the inner and outer solutions can be joined to form a composite solution uniformly valid to $O\left(\epsilon^{2}\right)$ everywhere in the channel. Because the thickness (in the $x$ direction) of the innex region is $O\left(\epsilon^{1 / 2}\right)$ and in case (1), the amplitude of the shock wave motion is $O(\epsilon)$, it is possible in case (1) to consider a stationary inner region. In the present case, the inner region thickness is again $O\left(\epsilon^{1 / z}\right)$, but as will be shown, the amplitude of the wave motion is $O(1)$, and a moving inner region must be accounted for. However, because $\tau=O\left(\epsilon^{-2}\right)$, it can be shown ${ }^{4}$ that the inner solutions are unchanged in form from those given in reference 1. The essential difference is that whereas in case (1) the shock motion is a perturbation about a steady state location and the relative velocity ahead of the shock wave is constant to first order, in the present case the shock wave may move throughout the channel, and the upstream fluid velocity relative to the shock depends on the shock position and is thus a function of time. Since to the order considered, no time derivatives remain in the differential equations for the inner region, the form of the solutions is the same in the two cases, the time dependence arising through the boundary conditions on the relative upstream velocity.

In view of the above remarks, it is seen that the general form of the composite solution holds either for case (1) or the present case; these solutions are repeated here, from reference 1 , for convenience.

$$
\begin{aligned}
& u=1+\epsilon u_{1}+\epsilon^{2} u_{2}+\ldots \\
& v=\epsilon^{2} v_{2}+\ldots \\
& P=1-\epsilon \gamma u_{1}-\epsilon^{2} \gamma u_{2}+\ldots \\
& \rho=1-\epsilon u_{1}-\epsilon^{2}\left(u_{2}+\left(\frac{\gamma-1}{2}\right) u_{1}^{2}\right)+\ldots \\
& \tilde{T}=1-\epsilon(\gamma-1) u_{1}-\epsilon^{2}(\gamma-1)\left(u_{2}+\frac{u_{1}^{2}}{2}\right)+\ldots
\end{aligned}
$$

where $\gamma=C_{p} / C_{v}$ is the ratio of specific heats, and where

$$
\begin{aligned}
& u_{1}= \pm \sqrt{\frac{2}{(\gamma+1)} f(x)+c_{w}} \\
& u_{2}=f^{\prime \prime} \frac{y^{2}}{2}+h_{x}+\zeta^{*} x^{*} \\
& v_{2}=f^{\prime} y+\zeta_{y}^{*}
\end{aligned}
$$

$\zeta^{*}=\frac{4 f_{0}^{\prime \prime}\left[(\gamma+1) C_{u}\right]^{1 / 2}}{\pi^{3}} \sum_{n=1}^{\infty} \frac{(-1)^{n}}{n^{3}} \cos (n \pi y)$

$$
\cdot \exp \left\{-\mathrm{n} \pi \mathrm{x}^{*} /\left[(\gamma+1) \mathrm{C}_{\mathrm{u}}\right]^{2 / 2}\right\}
$$


Finally, it is possible to calculate the shock

In equation (5a), $\mathrm{C}_{\mathrm{w}}$ is an arbitrary constant determined by the value of the velocity at the throat; i. e., if the flow is supersonic or subsonic there, $\mathrm{C}_{\mathrm{w}}>0$, while if the flow is one which accelerates from subsonic to supersonic speed and is therefore sonic at the throat, $C_{W}=0$. Also, in equations (5), $f^{\prime}=d f / d x$, etc., and $f_{o}^{\prime}$ is the value of $f^{\prime}$ at $x{ }_{s o}$ the zero order approximation to the shock-wave location, $x_{s}$, which is expanded as follows:

$$
x_{s}=x_{s o}(t)+\epsilon x_{s_{1}}(t)+\ldots
$$

For case (1), $x_{\mathrm{gO}}=$ constant, and in both cases, the $y$ dependence of $x_{s}$ occurs in higher order terms. In equations (5b) and (5c), the function $\zeta^{*}\left(x^{*}, y\right)$ is the contribution to the composite potential function from the inner solution. Upstream of the shock wave $\zeta^{*}=0$, and downstream $\zeta^{* *}$ is given by equation (5d). Finally, $C_{u}$ is the value of $u_{1}$ at $x$, evaluated upstream of the shock (upper sign in equation (5a)). In case (1), $\mathrm{C}_{\mathrm{u}}=$ constant, while in the present case, it is a function of time since, as will be seen, $x_{\text {so }}=x_{\text {so }}(t)$.

Before equations (5) can be evaluated, it is necessary to find $h_{x}$. The equation for $h_{x}$ is found from the next higher order solution in $v$, i. e., $v_{3}$, which satisfies the boundary condition that the flow remain tangent to the channel walls. For case (1) the differential equation for $h$ is ${ }^{1}$

$\frac{2 k_{1}}{(\gamma+1)} h_{t}+u_{1} h_{x}=-u_{1}\left(f^{t 1}+(2 \gamma-3) u_{1}^{2}\right) / 6+A(t)$

where the subscripts $t$ and $x$ represent partial differentiation. In reference 1 , a general numerical procedure for solution for $h_{x}$ was given, which permitted $u_{1}=u_{1}(x, t)$. In reference 3 , it was shown that if $u_{1}=u_{1}(x)$, the condition finally considered in reference 1 and considered here, then for case (1) $\left(\tau_{1}=\left(k_{1} \epsilon\right)^{-1}\right)$ the solution for $h_{x}$ can be written as follows :

$h_{x}=-\frac{1}{6}\left[f^{\prime \prime}+(2 \gamma-3) u_{1}^{2}\right]+\frac{C_{2}}{\dot{u}_{1}}+\frac{1}{u_{1}} G\left(t-t_{\ell}\right)$

$\mathrm{t}_{\ell}=\mathrm{t}_{\ell}(\mathrm{x})=\int_{\mathrm{x}}^{\mathrm{x}}\left(2 \mathrm{k}_{1} /(\gamma+1) \mathrm{u}_{1}(\xi)\right) \mathrm{d} \xi$

In equation (8a), $C_{2}$ is an arbitrary constant of integration set by boundary conditions and $G(t)$ is proportional to the oscillation in pressure impressed downstream of the shock wave, at $x=X$. That is, from equations $(4 c),(5 a)$ and $(5 b)$, it is seen that, as mentioned previously, the pressure varies with time in second order, and that at $x=X, G(t)$ is the time varying part of $h_{x}$ and thus of the pressure.

For the present case, where $\tau=O\left(\epsilon^{-2}\right), h_{t}$ is dropped from equation (7) and the solution is simply equation (8a) with $t_{\ell}=0$. That is, there is no lag between the impressed oscillations in pressure and velocity and the corresponding oscillations anywhere in that part of the flowfield affected by the impressed oscillations (between the shock position, $x$, and $X$ ). Signals travel upstream instantaneous $1 \mathrm{y}^{\mathrm{s}}$, compared to the period of the oscillation. position as a function of time. First, we consider the shock wave velocity, $u_{s}=d x_{s} / d T+O\left(\epsilon^{3}\right)$; $\left(\mathrm{V}=O\left(\epsilon^{7 / 2}\right) \text { is negligible }\right)^{2}$. Relative to the shock wave, the first order shock jump condition is, for transonic flow, $\left(u_{1}-u_{1 s}\right)_{d}=-\left(u_{1}-u_{1 s}\right)$, where $u_{1 s}$ is the first order absolute shock-wave velocity (i. e., $u_{s}=\epsilon u_{1 s}+\epsilon^{2} u_{2 s}+\ldots$ ) and where the subscripts $\mathrm{s}$ and d refer to conditions immediately upstream and downstream of the shock respectively. Now, $u_{1 u}$ and $u_{1 d}$ are given by equation ( $5 a$ ) with the upper and lower sign respectively, so $u_{d}=$ - $u_{k u}$, and from the shock relations, therefore, $\mathrm{u}_{\mathrm{ls}}=0$. Since the shock jump conditions a re not satisfied by $u_{2}$, it is clear that $u_{s_{2}} \neq 0$ and so $u_{s}=O\left(\epsilon^{2}\right)$. Therefore

$u_{s}=\frac{d x_{s}}{d t}=k \epsilon^{2} \frac{d}{d t}\left(x_{s o}+\epsilon x_{s 1}+\ldots\right)=O\left(\epsilon^{2}\right)$

and so $x_{\text {so }}=x_{s o}(t)$; this means that the lowest or dex termin $x$ sories with time so that the amplitude of the shock motion is O(1). For the shock motion in case (1), $\tau=O\left(\epsilon^{-1}\right)$ and $u_{s}=O\left(\epsilon^{2}\right)$, so that $x_{s o}=$ constant, $x_{s 1}=x_{s 1}(t)$, and the shock wave undergoes only small ${ }^{\text {s1 }}$ displacements from its equilibrium position ${ }^{1}$.

The governing equation for $\mathrm{x}_{\text {so }}(\mathrm{t})$, the first approximation to the instantaneous shock position, is derived by applying the mass conservation principle to a control volume containing (moving with) the shock wave. The change in entropy across the wave $\left(O\left(\epsilon^{3}\right)\right)$, is employed in writing the density downstream of the wave. Although variations in $\rho$ and $a$ up to third order must therefore be considered, the final result involves only second order terms, a result reported previously ${ }^{1,2,5}$. Details of the calculations are given in reference 4 , the resulting equation for $u_{\mathrm{s} z}=k d x_{s o} / d t$ is,

$\frac{4 k}{(y+1)} \frac{d x_{s o}}{d t}=h_{x d}+h_{x u}+\frac{f_{0}^{\prime \prime}}{3}-c_{u}^{2}$

This equation has exactly the same form as that given for case (1) (reference 1,) the only difference being that for case (1) $d x_{s 1} / d t$ is calculated; in the present case $C_{u}=C_{u}(t){ }^{s 1}$ whereas in case (1) $\mathrm{C}_{\mathrm{u}}=$ constant. For the specific problem considered here, where the flow upstream of the shock is steady and pressure oscillations are impressed upon the flow downstream of the shock at $x=X$, say, $h$ is given by equation (8a) evaluated at $\mathrm{x}=\mathrm{x}$, with $\mathrm{G}=0$. For a flow which is sonic at the throat (e.g., an accelerating flow), the case considered here, $C_{2 u}=0$ also. Again, subscripts $u$ and denote conditions immediately upstream and downstream of the shock wave, respectively. Equation (8a), evaluated at $x_{s o}$, is used for $h_{x d}$ (with $t_{1}=0$ since for the present calculation $\mathrm{xd}$ $\left.\tau=O\left(\epsilon^{\ell-2}\right)\right)$ : the value chosen for $C_{2 d}$ gives the steady state location for the shock wave, when $G(t)=0$. Thus, if the above mentioned relations for $h$ and $h$ are substituted into equation (10), one finds that

$\frac{4 \mathrm{k}}{(\gamma+l)} \frac{\mathrm{dx}}{\mathrm{dt}} \mathrm{s}^{1}=-\frac{1}{\mathrm{C}_{\mathrm{u}}}\left[\mathrm{C}_{2 \mathrm{~d}}+\frac{2 \gamma}{3} \mathrm{C}_{\mathrm{u}}^{3}+\mathrm{G}(\mathrm{t})\right]$ 
Hence, at the steady state shock location, where $\mathrm{dx} / \mathrm{dt}=\mathrm{G}(\mathrm{t})=0, \mathrm{C}_{2 \mathrm{~d}}=-2 \gamma \mathrm{C}^{3}(\mathrm{x}) / 3$, where $x_{s} o_{i s}$ now a constant. Thus, setting $C_{2 d}$ gives $\left.\mathrm{C}^{s} q_{\mathrm{x}}\right)$, which from equation (5a) means that the steady state value of $x$ can be calculated for a given wall shape. If we denote by $\mathrm{C}_{\text {uo }}$ the value of $C_{u}$ at this steady state location, then equation (11) can be written as follows:

$\frac{4 \mathrm{k}}{(\gamma+1)} \frac{\mathrm{dx}}{\mathrm{dt}}=\frac{1}{\mathrm{C}_{\mathrm{u}}}\left[\frac{2 \gamma}{3}\left(\mathrm{C}_{\mathrm{uo}}^{3}-\mathrm{C}_{\mathrm{u}}^{3}\right)-\mathrm{G}(\mathrm{t})\right]$

This equation thus gives the unsteady shock location measured from the steady flow location, for a given arbitrary impressed pressure oscillation represented by $\mathrm{G}(\mathrm{t})$.

Before analyzing the shock motion, it is of interest to note that it is possible to wiite a generalized solution, valid for either case (1), with $\tau$, = $O\left(\epsilon^{-1}\right)$, or for the present case, with $\tau=O\left(\epsilon^{-2}\right)$. Thus, if one replaces equation ( $8 \mathrm{~b})$ with the following generalized relation,

$$
t_{\ell}=\frac{1}{\tau \epsilon} \int_{X}^{x} \frac{2}{(\gamma+1)} \frac{d \xi}{u_{1}(\xi)}
$$

and equation (11) with the following equations

$$
\begin{gathered}
\frac{\mathrm{l}}{\tau \epsilon^{2}} \frac{4}{(\gamma+1)} \frac{\mathrm{dx}_{\mathrm{s}}^{+}}{\mathrm{dt}}=-\frac{1}{\mathrm{C}_{\mathrm{u}}}\left[\mathrm{C}_{2 \mathrm{~d}}+\frac{2 \gamma}{3} \mathrm{C}_{\mathrm{u}}^{3}+\mathrm{G}\left(\mathrm{t}-\mathrm{t}_{\ell \mathrm{o}}\right)\right](14 \mathrm{a}) \\
\mathrm{x}_{\mathrm{s}}=\mathrm{x}_{\mathrm{o}}+\mathrm{x}_{\mathrm{s}}^{+}(\mathrm{t})
\end{gathered}
$$

then the general solution is given by equations (4), (5), (8a), (13) and (14). In equations (14), $x$ is the steady state location of the shock, and $\mathrm{t}_{\ell \mathrm{O}}=\mathrm{t}_{\mathrm{f}}\left(\mathrm{x}_{\mathrm{o}}\right)$. It is seen that for $\tau=O\left(\epsilon^{-1}\right)$, then from equation (13), $t_{t}=O(1)$, and from equation (14a), $x_{s}^{+}=O(c)$, i.e., $\mathrm{x}_{\mathrm{s}}^{+}=\epsilon \mathrm{x}_{s_{1}}$. For $\tau=O\left(\epsilon^{-2}\right)$, then $\tau_{l}=O(c)$ and is negligible, and $x_{s}^{+}=O(1)$, so $x_{s}^{+}+x_{0}^{\ell}=x_{s o}$ The generalized solutions are particularly useful in making numerical calculations; it is easy to separate cases (1) and the present case asymptoti cally, but it is not easy to choose one case over the other in a given physical situation, i. e., with given numerical values of $\epsilon$ and $\tau$.

\section{Large Amplitude Shock Wave Motion}

As indicated previously, when $\tau=O\left(\epsilon^{2}\right)$ the shock wave motion resulting from pressure oscillations impressed downstream of the shock wave has an amplitude of order unity. As a result, there are conditions under which the shock will move upstream through the nozzle, disappear upstream, and then reappear as the downstream plenum pressure drops to the point where a shock wave in the channel is necessary to satisfy this instantaneous pressure requirement. The conditions for this occurrence and the subsequent shock wave motion depend in a complex manner upon the amplitude of the forcing function, $G$, the steady state conditions about which the oscillations occur, represented by $C_{2 d}$, the wall shape $f(x)$, and the numerical value of the time constant, represented by $k$.
The equation which governs the shock motion is equation (11) or equation (12), where, since $\mathrm{x}_{\mathrm{s}}=\mathrm{x}_{\mathrm{so}}+\mathrm{O}(\epsilon)$, then to the order considered here, $\mathrm{x}_{\mathrm{S}}$ and $\mathrm{x}_{\mathrm{s}} \mathrm{are}$ interchangeable. It is interesting to note that although signals from the impressed disturbances reach the shock wave instantaneously in a first approximation, the shock wave does not respond instantaneously. The shock velocity is finite, and indeed there is a lag between the impressed disturbance $G(t)$ and the resulting shock velocity $\mathrm{dx}_{\mathrm{so}} / \mathrm{dt}$. In equation (12), for example, it is seen that the term $\left\langle\mathrm{C}_{\text {uo }}^{3}-\mathrm{C}_{\mathrm{u}}^{3}\right\rangle$ always has a sign such that its effect is to cause the shock to move toward the equilibrium or steady state position. On the other hand, $G(t)$ is a forcing function which changes sign periodically. The result is a shock motion which lags $G(t)$.

It is clear from equations (11) or (12) that singularities occur as the shock wave approaches the throat and $\mathrm{C}_{\mathrm{u}} \rightarrow 0$. The behavior of integral curves which cross the $x_{\text {u }}=0$ axis can be found for a sinusoidal $G(t)$, for example, by writing equation (11) for $x_{s_{0}}$ (and thus $C_{u}$ ) small compared to unity and for $\left|t-t_{0}\right| \ll 1$, where to is the value of $t$ at which the bracket on the right hand side of equation (11) goes to zero at the throat $x_{\text {so }}=0$. Thus, if, for example,

then

$$
G=G_{0} \sin b t
$$

$$
\sin b t_{0}=-C_{2 d} / G_{0}
$$

and equation (11) becomes, for $x_{\text {so }} \ll 1$ and $\left|\mathrm{t}-\mathrm{t}_{\mathrm{o}}\right| \ll 1$,

$$
\begin{gathered}
\frac{d x_{s o}}{d t}=-\frac{(\gamma+1)}{4 k}\left(b G_{0} \cos b t_{0}\right)\left(\frac{t-t_{0}}{C_{u}}\right) \\
\cos b t_{0}= \pm \sqrt{1-\left(C_{2 d} / G_{o}\right)^{2}}
\end{gathered}
$$

where, again, $C_{u}=u_{1 u}\left(x_{s o}\right)$ is the value of $u_{1}$ at $x=x_{s}(t)$, upstream of the shock, and where $\mathrm{C}_{\mathrm{u}}^{3} \ll\left|\mathrm{t}-\mathrm{t}_{\mathrm{o}}\right|$. A typical wall shape and the corresponding solution to equation (17a) are, in the neighborhood of the throat,

$$
f(x)=a x^{2}
$$

$x_{\text {so }}^{2}=-\frac{(\gamma+1)^{3 / 2}}{2^{5 / 2} k} \frac{b}{\sqrt{a}} G_{0}\left(\cos b t_{o}\right)\left(t-t_{0}\right)^{2}$

Thus, if $\cos b t_{p}>0$, the point $\left(0, t_{0}\right)$ is a center and the integral curves (ellipses) in the neighborhood of this center cross $x_{s}=0$ with an infinite slope. On the other hand, if $\cos b t_{0}<0$, the integral curves in the neighborhood of $\left(0, t_{0}\right)$ are hyperbolae, the point being a saddle point, and the two integral curves pass through the point $\left(0, t_{0}\right)$ with slopes

$$
\frac{d x_{s o}}{d t}= \pm\left\{\frac{(y+1)^{3 / 2}}{2^{5 / 2} k} \frac{b G_{o}}{a^{1 / 2}}\left|\cos b t_{0}\right|\right\}^{1 / 2}
$$

An understanding of the possible shock motions may be gained by analyzing the integral curves which pass through the saddle points. The three possible configurations for these curves are 
sketched in figure 2. In these sketches, the arrows indicate the direction the solutions must follow as time increases. In figure $2 a$, conditions are such that the integral curves entering the saddle point originate from a particular $x_{s o}=x_{o}$ at $t=0$. Those leaving the saddle begin to rise, then reverse their directions and cross the time axis with vertical slope at some point between the center (indicated by -) and the next saddle point (indicated by $x$ ). Other integral curves are sketched also, as dotted lines. As indicated in the sketch, the paths traced by the integral curves are repetitive. In figure $2 \mathrm{c}$, the opposite situation exists; the integral curves entering the saddle point begin on the abscissa, between a saddle and a center, with an infinite slope and then change direction and enter the next saddle point. Those curves leaving the saddle never return to the axis $x_{s o}=0$, but asymptotically approach a'single periodic curve (for a given $\mathrm{C}_{2 \mathrm{~d}}$ ). Those curves which originate with an $\mathrm{x}_{\mathrm{so}}$ greater than any $\mathrm{x}_{\mathrm{s}}$ on this periodic curve will approach the periodic curve asymptotically from above. This periodic curve is nearly symmetric about $x_{0}$, the steady $s$ tate value of $x_{\mathrm{so}}$. In the dividing case, shown in figure $2 \mathrm{~b}$, the curves entering and leaving the saddle points are the same curve.

The integral curve map obtained in any given case depends upon $C_{2 d}, k, G(t)$, and the wall shape, $f(x)$. Although general solutions from which a general criterion for the dividing condition (figure 2b) could be derived are not available, an approximate result can be found for $G$ as given in equation (15) and $f(x)$ as in equation (18a). Then, equation (11) becomes

$K x_{\text {so }} \frac{d x_{\text {so }}}{d t}=-C_{2 d}-\Gamma x_{\text {so }}^{3}-G_{o} \sin b t$

$K=\frac{2^{5 / 2} \mathrm{k} / \sqrt{\mathrm{a}}}{(y+1)^{3 / 2}}$

$$
\Gamma=\frac{2 y}{3}\left(\frac{2 a}{y+1}\right)^{3 / 2}
$$

and the slopes of the integral curves at the saddle points are given by equation (19), with $t_{0}$ and $G_{0}$ related as in equation (16). Now, if it is assumed that the integral curve which passes through the saddle point at $b t=b t$ and also through the next saddle point at $b t=b t_{0}^{o}+2 \pi$ (e.g. , see figure $2 b$ ), is approximately symmetric about $b t=b t_{o}+\pi$, then the maximum value of $x_{50}$ is, from equation (20a),

$$
\left(\mathrm{x}_{\mathrm{so}}\right)_{\mathrm{m}}=\left(-2 \mathrm{C}_{2 \mathrm{~d}} / \Gamma\right)^{1 / 3}
$$

Next, if equation (20a) is integrated first over one period (e.g, bt $=b t_{0}$ to $\left.b t=b t_{0}+2 \pi\right)$ and then over $a$ half period, then since $x_{S o}=0 a t b t_{o}$ and $b t_{o}+2 \pi$ and $x_{s o}=\left(x_{s o}\right)_{m}$ at $b t=b t_{o}+\pi$, one finds the following relations

$$
\begin{gathered}
0=C_{2 d}+r \int_{0}^{1} x_{s o}^{3} d \tilde{t} \quad \tilde{t}=b\left(t-t_{0}\right) / 2 \pi \\
\frac{K\left(x_{s 0}\right)_{m}^{2}}{2}=-2 \frac{G}{b} \cos b t_{0} .
\end{gathered}
$$

where, in equation (22c), advantage has been taken of the fact that the integral of $x_{B 0}^{3}$ over half a period is half the integral over a full period because of the symmetry of $x_{\mathrm{SO}}$. Substituting for cos bt using equation (16), one finds from equation $(22 \mathrm{c})$ the following relation for $G_{0}$, for the special case (figure $2 \mathrm{~b}$ ):

$$
\left(G_{o}^{2}-C_{2 d}^{2}\right)^{1 / 2}=b K\left(x_{s o}\right)_{m}^{2} / 4
$$

where $\mathrm{K}$ is given in equation (20b). Although this equation is useful in setting a first approximation for $G_{O}$, a more accurate result may be found by taking into account the fact that the integral curve in question is not in fact symmetric, but is slightly asymmetric. In this calculation, it is necessary to employ an approximate form for $x_{\text {so }}(t)$; a cubic equation of the following form suffices:

$$
x_{\text {so }}=C_{1} \tilde{t}(1-\tilde{t})+C_{2} \tilde{t}\left(1-\tilde{t}^{2}\right)
$$

Now, at $x_{\text {so }}=\left(x_{\text {so }}\right)^{m}$, where $d x_{\text {so }} / d t=0, \tilde{t}$ is defined as $\begin{array}{r}\tilde{\mathrm{t}}_{\mathrm{m}} \text {, where } \\ \tilde{\mathrm{t}}_{\mathrm{m}}=\frac{1}{2}+\delta\end{array}$

Also, it is assumed that $\delta$ is numerically small enough that terms involving $\delta^{2}$ may be ignored. Then, from equation (20a) evaluated at $x_{s o}=\left(x_{s o}\right)_{m}$, equation (22a) with equation (24) used in the evaluation of the integral, equation (20a) integrated over one half period $\tilde{t}=0$ to $\tilde{t}=1 / 2$ ) with equation (24) used in integrating the $x_{s o}^{3}$ term, $\left(x_{s o}\right)_{m}$ evaluated using equation (24), and equation (25), one can derive the following relations for $\left(x_{\mathrm{so}}\right)_{\mathrm{m}}, \delta$, and finally, $G_{0}$.

$$
\begin{aligned}
& \left(x_{s o}\right)_{m}=\left(-35 C_{2 d} / 16 \Gamma\right)^{1 / 3}, \delta=3 C_{{ }_{2 d}} / 8 \pi b K\left(x_{s o}\right)_{m}^{2} \\
& \left(G_{o}^{2}-C_{2 d}^{2}\right)^{1 / 2}=\frac{b K\left(x_{s o}\right)_{m}^{2}}{4}-\delta \pi\left(0.207 \Gamma\left(x_{s o}\right)_{m}^{3}-C_{2 d}\right)
\end{aligned}
$$

where, again, $K$ and $\Gamma$ are defined in equations $(20 \mathrm{~b}, \mathrm{c})$.

Example calculations of the integral curves through the saddle points, with the sinusoidal forcing function given in equation (15) and with parabolic walls as in equation (18a), are shown in figure 3 ; the first approximation to the special value of $G_{O}$ for case (b), calculated using equation (26c), must be modified using trial and error. The calculations were carried out by numerically integrating equation (20a), using equation (19) to find an initial condition near $x_{\text {so }}=0$. In the calculation, $\mathrm{b}=2, \mathrm{k}=1, \mathrm{a}=(\mathrm{y}+1) / 2=1.2, \mathrm{x}_{0}=1.5, \mathrm{c}_{\mathrm{u}}=$ $(2 f(x) /(y+1))^{1 / 2}$, and $C_{2 d}=-2 y C_{u o}^{3} / 3$, where $x_{0}$ is the steady state value of $x_{s o}$. In figure 3 , the letters $a, b$, and $c$, refer to the corresponding cases shown in figure 2. In each case, only the curves through one saddle point are shown; the repetitive nature of the curves at each saddle point is not shown, for clarity. It should be noted that the value $t_{0}$ in figure 3 , referring to the location of a saddle point, is different for each case. The centers, which also occur at different values of $t$ for 
each case, are noted in figure 3. With the parametric values given above, it was found that for the special case, shown in figure $2 \mathrm{~b}$, the special value for $G_{0}$ was, from equation $(23),\left(G_{o}\right)_{s p}=4.33$ and from equation $(26 \mathrm{c}),\left(G_{0}\right)_{s p}=4.77$. The value which gives accurate results (figure 3 ) is

$$
\left(G_{\mathrm{o}}\right)_{\mathrm{sp}}=4.968
$$

Thus, equation (26c) is helpful in giving a relative. ly accurate ( $4 \%$ exror) first guess for $\left(G_{o}\right)_{s p}$; in another case, with all other parameters the same, but with $x_{0}=0.75$, it was found that equation $(26 \mathrm{c})$ gave an estimate with an error of $6 \%$. The curves labeled $a$ and $c$ in figure 3 were calculated using $G_{0}=5.5>\left(G_{o}\right)_{s p}$ and $G_{o}=4<\left(G_{o}\right)_{s p}$, respectively. In each of these cases, curves entering and leaving the saddle point at $t-t_{o}=0$ are shown, the behavior in each case following that sketched in the corresponding part of figure 2. The solutions shown in figure 3 are for very simple (parabolic) wall shapes. There appears to be no simple way of predicting $\left(G_{0}\right)_{s p}$ for more complicated wall shapes; in general, it is necessary to integrate numerically along an integral curve leaving a saddle point to see which case occurs for the given parameters. Examples are shown later.

With the mathematical behavior of the integral curves through saddle points understood, it is possible to interpret the physical behavior of the shock wave in each case. Referring to figure $2 a$, for any initial condition which does not lie on an integral curve entering a saddle point (two are illustrated by circles in figure $2 a$ ), the shock passes through the throat and disappears upstream. This is seen by following the integral curve in question as time increases: $x_{\text {so }}$ goes to zero, for any initial condition, between a center and a saddle point. As time increases, then, a saddle point occurs at $x_{0}=0$, and an integral curve rises from the saddle point in the direction of increasing $t$. This means that the back pressure has decreased to the point where a shock wave must form in the channel in order to satisfy the instantaneous pressure requirements. This is seen by writing the pressure at $x=X$, using equations $(4 c),(5 b)$, and (8a) with $t_{\ell}=0$; for $f^{\prime \prime}(X)=0$,

$$
\begin{aligned}
P_{b}=1-\epsilon \gamma u_{1}(X) & +\epsilon^{2} \gamma\left[\left(\frac{2 \gamma-3}{6}\right) u_{1}^{2}(X)-\frac{C_{2 d}+G(t)}{u_{1}(X)}\right] \\
& +\ldots
\end{aligned}
$$

where $u_{1}(X)<0$. From equation (28), it is seen that the conditions for the back pressure to be that which gives the subsonic solution for $0<x<x$, with sonic pressure at the throat is

$$
C_{a_{d}}+G(t)=0
$$

But this condition, for the case where $G(t)$ (and hence the pressure) is decreasing, is precisely the condition for the saddle point, as exemplified by equations (15) and (16) and the discussion following these equations. The fact that this back pressure requirement must be satisfied instantaneously by a shock forming at the throat is a result of the fact that there is no time lag in the solutions for the velocity, pressure, etc. As a result, then, the proper behavior for the shock, after it disappears, is to reappear at the time as sociated with the first saddle point after its disappearance. I't then follows the path given by the integral curve through the saddle point and so dis appears again, forms again at the throat at the following saddle, etc. Thus, no matter what the initial condition is, the resulting shock motion is associated with the integral curves leaving the saddle points, as shown in figure $4 \mathrm{a}$. For the periods of time between the disappearance and reappearance of a shock wave, the flow is subsonic throughout the channel. If the initial condition should lie on an integral curve entering the saddle point, the shock moves to the throat and moves away again on the integral curve leaving the saddle point. Thereafter, its motion is the same as that shown in figure $4 \mathrm{a}$.

Referring now to the dividing case shown in figure $\mathrm{Zb}$, it is seen that there are several different possibilities for the shock motion, depending on the initial condition, again indicated by circles. If the initial condition lies outside the integral curves through the saddle points, the shock position merely oscillates with time, never going through the throat. If the initial conditions lies beneath the integral curves through the saddle points, the shock moves upstream, passes through the throat and disappears; then for the same reasons mentioned in the previous case, it forms at the throat at the time corresponding to the first saddle point after its disappearance. It then follows the integral curves through the saddle points, so that thereafter, it just moves to the throat and never passes upstream; this motion is illustrated in figure $4 \mathrm{~b}$. If the initial condition should lie on an integral curve through a saddle point, the shock position is completely described by integral cur ves through the saddle points; the shock never moves upstream of the throat.

Finally, referring to figure $2 \mathrm{c}$, there are again several possible initial conditions. If the initial condition lies above the integral curve entering the saddle point, the shock motion approaches a periodic form, never reaching the throat. If it lies on an integral curve below the curve entering the saddle point, it moves upstream through the throat and disappears, forms at the throat at the time corresponding to the first saddle point after its disappearance, and then moves away from the throat and approaches a periodic motion, never approaching the throat again. This motion is shown in figure $4 c$. Finally, if the initial condition should lie on the integral curve entering the saddle, the shock wave moves to the throat, moves away immediately on the integral curve leaving the saddle point, and approaches the same periodic motion mentioned above.

The numerical examples shown so far (e.g. figure 3) have been for simple wall geometries for which it is possible to derive an approximate relationship between the parameters for the special dividing case shown in figure 2. (Equation 
26c). For general geometries, it is necessary to integrate equation (11) numerically along the integral curves leaving the singularity, using equation (17a) to find starting values near $x_{\mathrm{so}}=0$, to find which case holds. Examples of such calculations, for more complicated wall shapes, are shown in figures 5 and 6 ; in these calculations $f(x)$ is as follows :

$$
\begin{aligned}
f(x) & =18 x^{2} / 13 & & x<1 \\
& =27(x-2)^{4} / 13+48(x-2)^{3} / 13+3 & & 1 \leq x \leq 2 \\
& =3 & & x>2
\end{aligned}
$$

Figure 5 shows calculations made for $\mathrm{C}_{2 \mathrm{~d}}=0$, that is, for the case where the steady state solution is that for which the flow goes through sonic velocity at the throat but is subsonic thereafter, with no shock waves. Clearly, the unsteady motion is that illustrated in figure 2a. In figure 6, two examples are shown in which the only parameter varied is the steady state shock position, $x_{0}$. Referring to the integral curves through the first saddle points, it is seen that for $x_{0}=1.5$, the situation is that illustrated in figure $2 c$, while for $x_{0}=0.75$, it is that illustrated in figure $2 \mathrm{a}$. Also shown in figure 6 are the solution curves from the initial condition to the point where the shock pas ses through the throat. With these two curves and those leaving the first saddle point, one can find then the resulting shock wave motions corresponding to figures $4 a\left(x_{0}=0.75\right)$ and $4 \approx\left(x_{0}=1.5\right)$.

\section{Conclusions}

The above examples illustrate the remarkably varied shock wave motions governed by the simple first order nonlinear equation (11). Moreover, they illustrate the well known fact that in transonic channel flows, small changes in downstream presm sures can cause large local changes in pressure by changing the location of the shock; in these examples, the pressure jump across the shock is $O(\epsilon)$, and the position is governed by changes in back pressure $O\left(\epsilon^{2}\right)$. Finally, they show that large changes in shock position can result from small changes in back pressure; i. $\epsilon$. , for $\Delta P_{b}=O\left(\epsilon^{2}\right), \Delta x_{s}=O(1)$. The solutions presented allow relatively simple calculations of shock positions to be made for transonic flows in symmetric channels with arbitrary wall shapes and arbitrary oscillations in back pressure. The extension to asymmetric channels, still with radius of curvature $O\left(\epsilon^{-2}\right)$ is not difficult, and it appears that these results may have application to inlet buzz and to flutter problems in turbomachinery.

\section{References}

1. Richey, G. K., and Adamson, Jr., T. C., "Analysis of Unsteady Transonic Channel Flow with Shock Waves," AIAA Journal, 14, August 1976. pp. 1054-1061.

2. Chan, J. S. -K., and Adamson, Jr., T. C., "Unsteady Transonic Flows with Shock Waves in an Asymmetric Channel, "Submitted for publication.
3. Messiter, A. F., and Adamson, Jr, , T. C., "As ymptotic Solutions for Nonsteady Transonic Channel Flows," Symposium Transsonicum II, Eds., K. Oswatitsch and D. Rues, Springer-Verlag, 1976, pp. 41-48.

4. Adamson, Jr., T. C., and Liou, M. S., "Unsteady Motion of Shock Waves in Two Dimensional Transonic Channel Flows, " Report UM 014534-F, June, 1977.

5. Messiter, A. F., and Adamson, T. C., Jr. "On the Flow Near a Weak Shock Wave Downstream of a Nozzle Throat, " J. Fluid Mech., vol. 69, 1975, pp. 97-108.

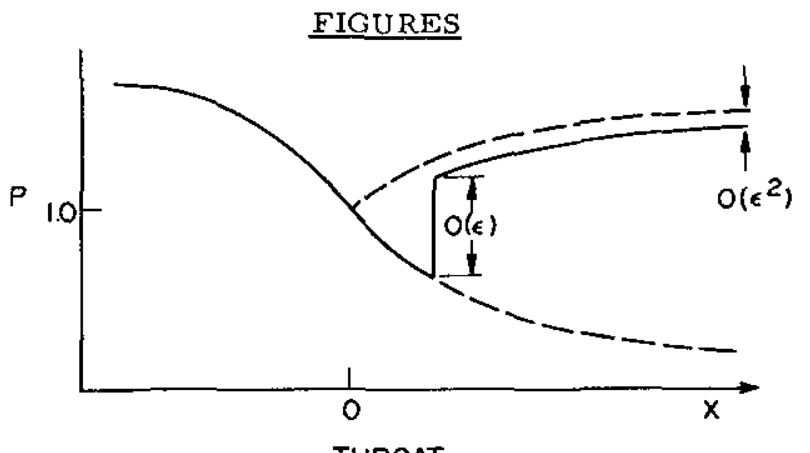

Fig. 1. Pressure distribution in nozzle with accelerating flow. ---shockless flow; flow with a shock wave.

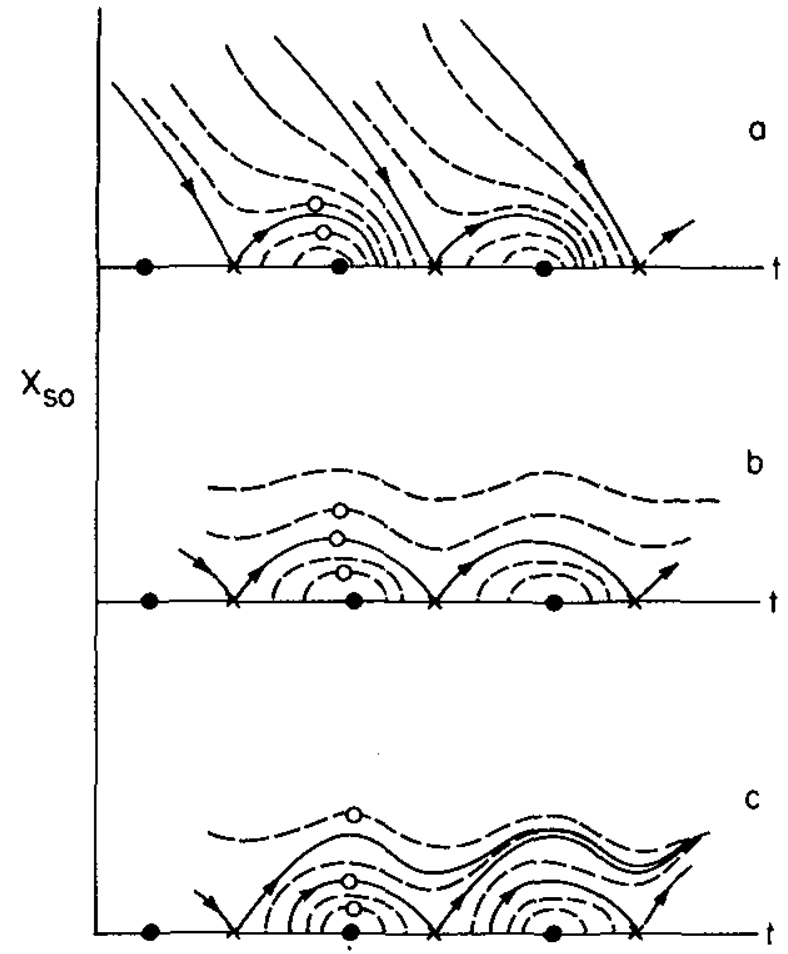

Fig. 2. Sketch of possible configurations for integral curves through saddle points (solid lines); othe $r$ integral curves indicated by dashed lines. - center; $x$ saddle point. (a) Integral curves leaving $x$ reach time axis before next $x$. (b) Integral curves leaving $x$ reach time axis at next $x$. (c) Integral curves leaving $x$ never return to time axis. 


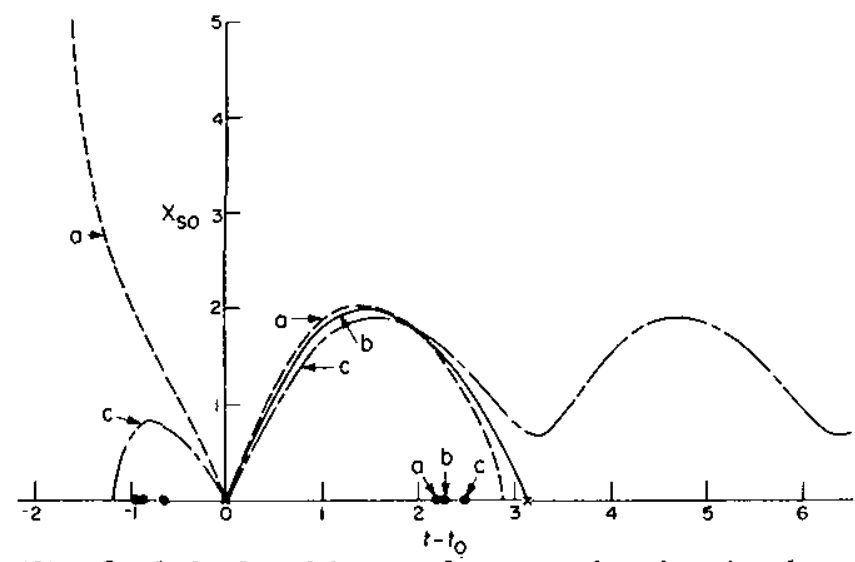

Fig. 3. Calculated integral curves for the simple case represented by eqs. (15), (18a), (26), and (20a), with $\mathrm{b}=2, \mathrm{k}=1, \mathrm{a}=(\mathrm{\gamma}+\mathrm{l}) / 2=1.2, \mathrm{x}_{0}=1.5$, and $\mathrm{C}_{2 \mathrm{~d}}$ $=-2 \gamma \mathrm{C}_{\mathrm{uo}}^{3} / 3$, illustrating the three cases sketched in fig. 2; curves labeled a(---), b(-), and $\mathrm{c}(---)$ refe $r$ to the corresponding cases in fig. $2 . x_{0}$ is the steady state value of $\mathrm{x}_{\mathrm{so}}$. -cente $\mathrm{r} ; \mathrm{x}$ saddle point.

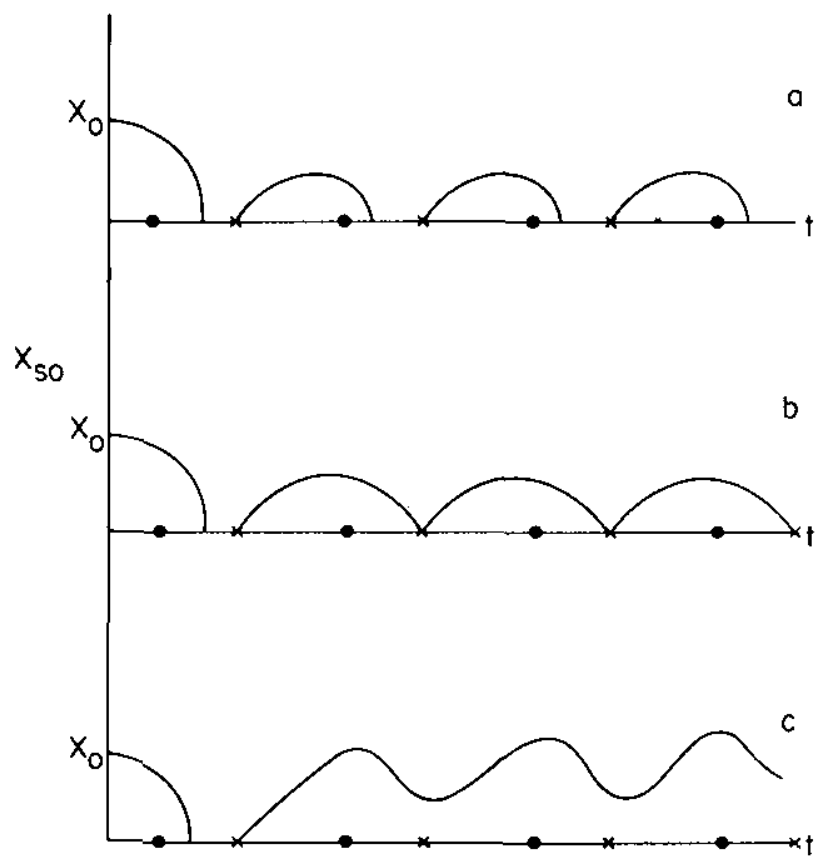

Fig. 4. Sketches of shock wave motion when the amplitude of the impressed pressure oscillation is large enough to drive the shock wave upstream of the throat, for each of the three cases shown in fig. 2 ; cases labeled a, b, and $c$ refer to the corresponding cases in fig. $2 . x_{0}$ is the initial, steady state, condition for the shock position.

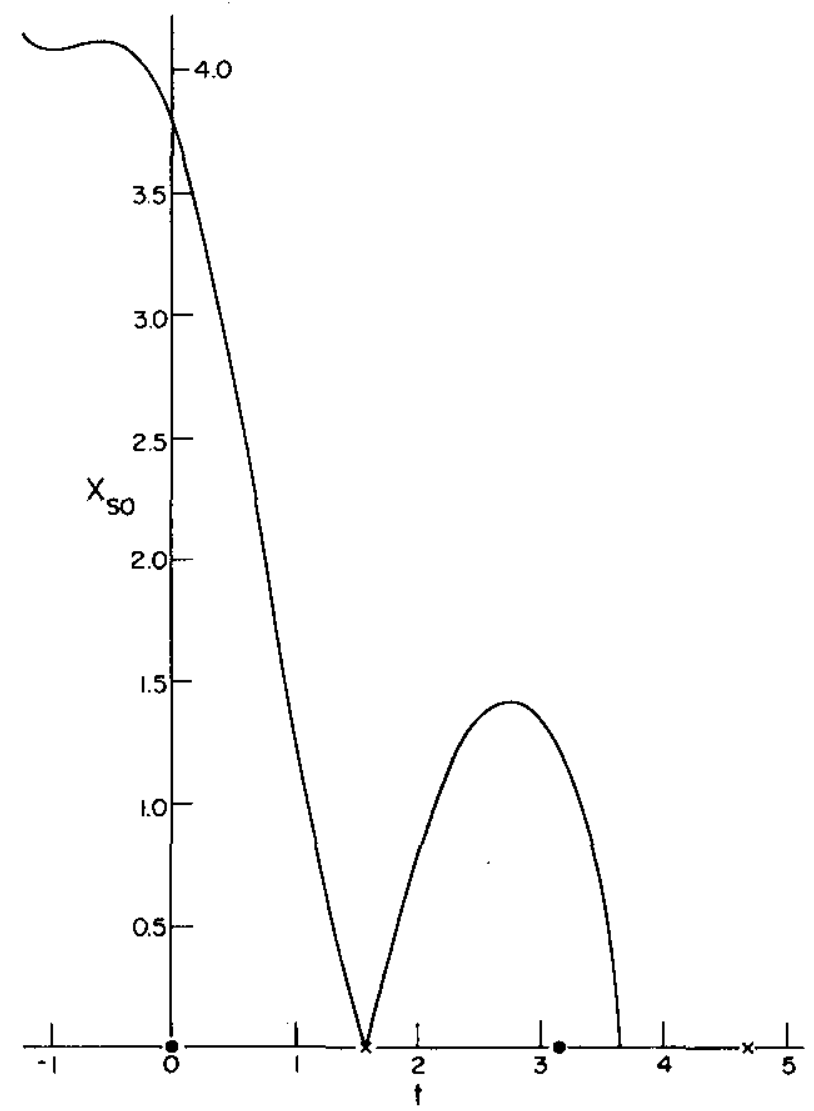

Fig. 5. Calculated integral curves through the saddle point illustrating the case sketched in fig. $2 \mathrm{a}$, for $C_{2 d}=0, G=4 \sin 2 t, \gamma=1.4, T=100, \epsilon=0.1$, $\mathrm{C}_{\mathrm{w}}=0$, and $f(x)$ as given in eqs. (30). Solutions found by numerically integrating eq. (11).

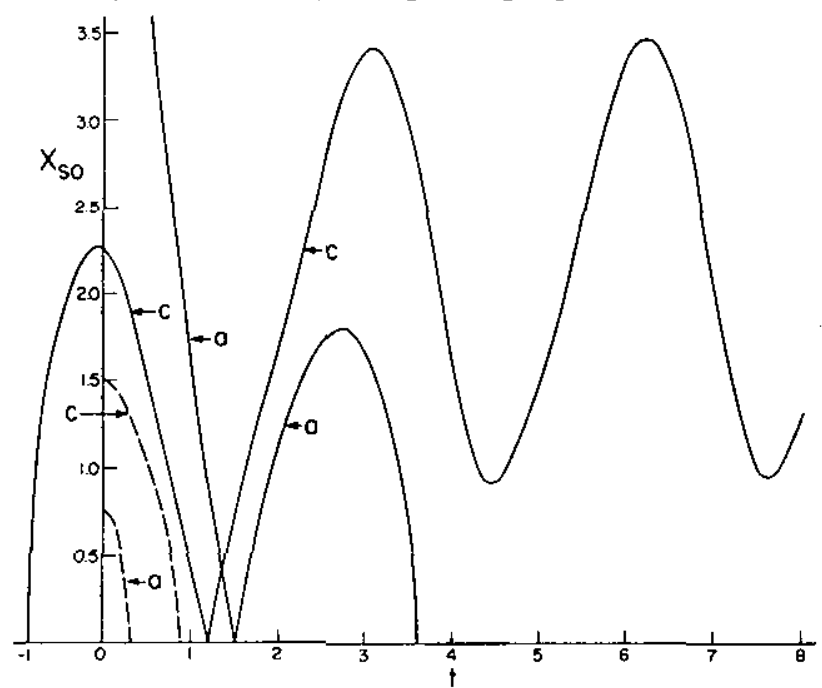

Fig. 6. Calculated integral curves through the saddle points (solid lines) and from the initial condition to the time axis (dotted lines) for two values of $x_{0}$ and thus $C_{2 d}$. For curves marked a, corresponding to the case sketched in fig. $2 \mathrm{a}, \mathrm{x}_{0}=1.5$; for curves marked $c$, corresponding to the case sketched in fig. $2 \mathrm{c}, \mathrm{x}_{\mathrm{o}}=0.75$. For each case, $\mathrm{G}=4.5 \sin 2 \mathrm{t}, \gamma=1.4, \tau=150, \epsilon=0.1, \mathrm{C}_{\mathrm{w}}=0$, and $f(x)$ is as given in eqs. (30). 